\title{
Covering the Plane with Copies of a Convex Disk
}

\author{
D. Ismailescu \\ Courant Institute, New York University, \\ 251 Mercer Street, New York, NY 10012, USA
}

\begin{abstract}
We prove that for every convex disk $\mathcal{C}$ in the plane there exists a double-lattice covering of the plane with copies of $\mathcal{C}$ with density $\vartheta \leq 1.2281772$. This improves the best previously known upper bound $\vartheta \leq 8 /(3+2 \sqrt{3}) \approx 1.2376043$, due to Kuperberg, but it is still far from the conjectured value $\vartheta=2 \pi / 3 \sqrt{3} \approx 1.2091993$.
\end{abstract}

\section{Introduction}

Given a convex disk (i.e., compact convex set) $\mathcal{C}$ in the plane, let $\vartheta(\mathcal{C})$ denote the minimum density of a covering of the plane with copies of $\mathcal{C}$. Further, let $\vartheta=\max \vartheta(\mathcal{C})$, where the maximum is taken over all convex disks on the plane.

According to a classical conjecture $\vartheta=2 \pi / 3 \sqrt{3}$. It is known that $\vartheta(\mathcal{C}) \leq 2 \pi / 3 \sqrt{3}$ holds for every centrally symmetric convex disk $\mathcal{C}$, with equality if and only if $\mathcal{C}$ is a circular disk or an ellipse (see [1], [2], [4], and [5]).

For general convex disks, the best previously known upper bound was established by Kuperberg [3]: $\vartheta \leq 8 /(3+2 \sqrt{3})$.

In Kuperberg's proof the following notion played a key role. A convex hexagon is called a $p$-hexagon if it has two parallel edges of the same length, opposite to each other (in the sense that they are separated by exactly two edges). It is easy to see that the plane can be tiled with congruent copies of any $p$-hexagon in a double-lattice fashion (see Fig. 1).

Thus, given a $p$-hexagon $H$ inscribed in a convex disk $\mathcal{C}$, we can generate a doublelattice covering of the plane with copies of $\mathcal{C}$ whose density is $\vartheta^{\prime}=\operatorname{area}(\mathcal{C}) / \operatorname{area}(H)$. Therefore, finding an inscribed $p$-hexagon with large area results in a low density (double-lattice) covering with congruent copies of $\mathcal{C}$.

Let $\mathcal{C}$ be a convex disk and let $\theta$ be an arbitrary direction (see Fig. 2). Let $t_{\theta}$ and $t_{\theta}^{\prime}$ be the tangents to $\mathcal{C}$ that are parallel to $\theta$. These lines touch the boundary at $A$ and $A^{\prime}$ (if $t_{\theta} \cap$ bd $(\mathcal{C})$ contains more than one point-and this is the case when the boundary 


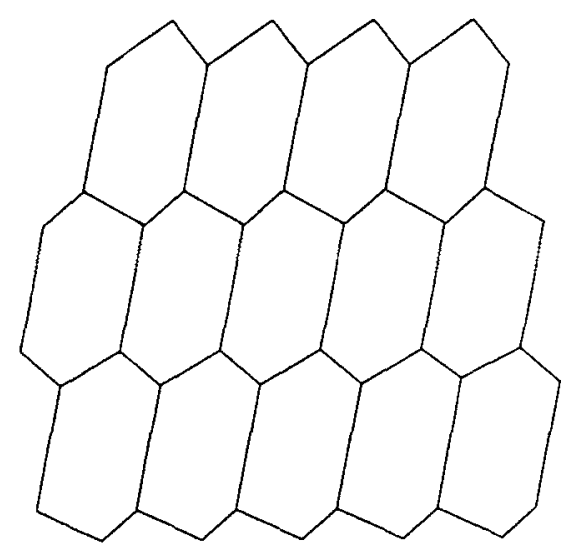

Fig. 1

of $\mathcal{C}$ contains a segment parallel to $\theta$-then let $A$ denote an arbitrary point from the intersection). Consider now the tangents to $\mathcal{C}$ that are parallel to $A A^{\prime}$ and denote by $B$ and $B^{\prime}$ the points where these lines touch the boundary. Now, for every $0 \leq l \leq\left|A A^{\prime}\right|$ we can find points $M, N, P$, and $Q$ on the boundary of $\mathcal{C}$ so that $M N\|P Q\| A A^{\prime}$ and $|M N|=|P Q|=l$. We obtain two $p$-hexagons inscribed in $\mathcal{C}: H_{1}(\theta, l)=B M Q B^{\prime} P N$ and $H_{2}(\theta, l)=M A Q P A^{\prime} N$. We say that these two $p$-hexagons belong to direction $\theta$. Clearly, there are infinitely many $p$-hexagons belonging to the same direction because $l$ can be chosen arbitrarily between 0 and $\left|A A^{\prime}\right|$. Kuperberg's results can be formulated as follows.

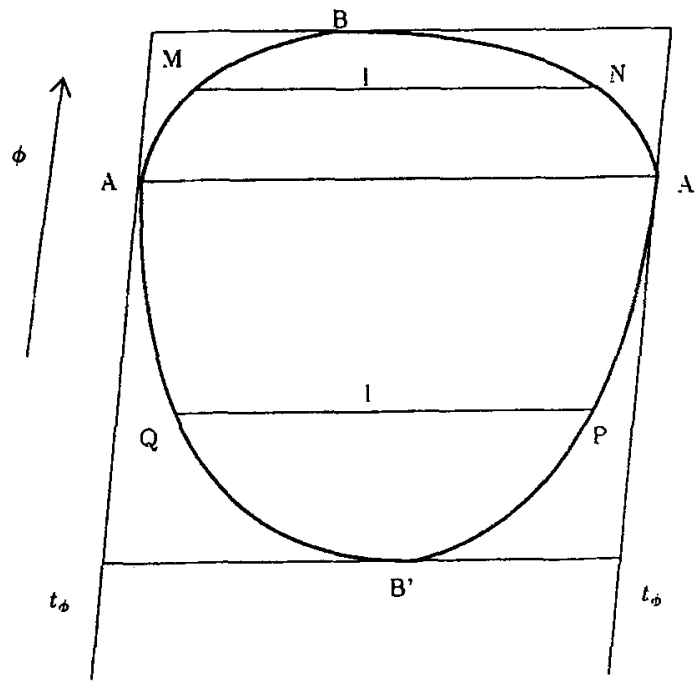

Fig. 2 


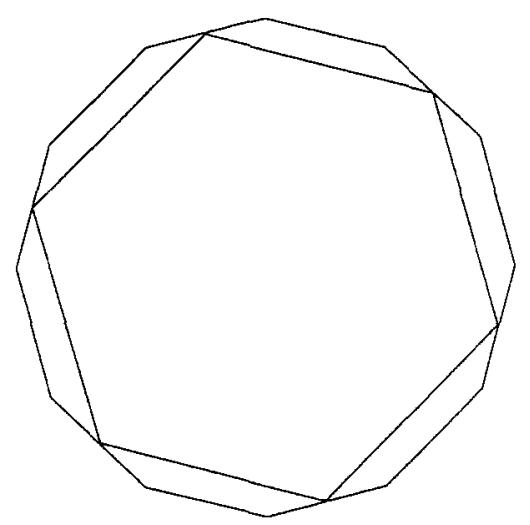

Fig. 3

Theorem A [3]. For every convex disk $\mathcal{C}$ and for every direction $\theta$, there exists a $p$ hexagon $H_{0}$ belonging to direction $\theta$ such that $\operatorname{area}(\mathcal{C}) / \operatorname{area}\left(H_{0}\right) \leq 8 /(3+2 \sqrt{3})$.

The above result is best possible in the following sense.

Theorem $\mathbf{B}$ [3]. There exist a convex disk $\mathcal{C}_{0}$ and a direction $\theta_{0}$ such that every $p$ hexagon $H$ belonging to the direction $\theta_{0}$ satisfies area $\left(\mathcal{C}_{0}\right) / \operatorname{area}(H) \geq 8 /(3+2 \sqrt{3})$.

This is the case when $\mathcal{C}_{0}$ is a regular dodecagon and $\theta_{0}$ is the direction of one of its edges (see Fig. 3).

The main result of this paper is the following.

Theorem. For every planar convex disk $\mathcal{C}$ there exist a direction $\theta(\mathcal{C})$ and a p-hexagon $H$ belonging to this direction that satisfies

$$
\vartheta=\frac{\operatorname{area}(\mathcal{C})}{\operatorname{area}(H)} \leq \frac{4\left(\sqrt{2}-2 \sin x_{0}\right)}{4+\sqrt{2}+2 \sin x_{0}}+\frac{4 x_{0}}{x_{0}+\sqrt{2}}=1.2281771 \ldots,
$$

where $x_{0}=0.2594296 \ldots$ is the real root of the equation $\cos x-x=\sqrt{2} / 2$.

\section{The Main Construction}

Let $\mathcal{C}$ be a convex disk and let $T W U V$ be the quadrilateral of maximum area inscribed in $\mathcal{C}$ (see Fig. 4). Let $d_{T}$ denote the line passing through $T$ and parallel to $W V$. Obviously, $d_{T}$ is tangent to $\mathcal{C}$.

We obtain (making the same constructions for points $W, U$, and $V$ ) a parallelogram circumscribed about $\mathcal{C}$ so that $T U$ and $V W$ are parallel to the edges of this parallelogram. 


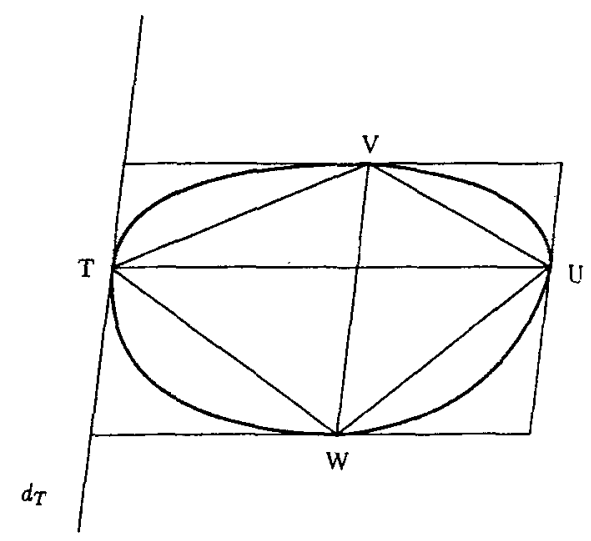

Fig. 4

Applying an appropriate affine transformation if necessary, we can assume that this parallelogram is a unit square.

Moreover, since $T W U V$ is the quadrilateral of maximum area inscribed in $\mathcal{C}$, it follows that every quadrilateral inscribed in $\mathcal{C}$ has area at most $\frac{1}{2}$. This is used later to prove Lemma 3.

Let $n$ be a positive integer and let $\alpha=\pi / 4 n$. For every $k \in\{1,2, \ldots, 2 n-1\}$ take a pair of segments of length $L_{k}=\sin k \alpha$, that are parallel to $T U$, and whose ends are both on the boundary of $\mathcal{C}$. Let these points be as indicated in Fig. 5. Hence, we have $A_{k} B_{k}\left\|C_{k} D_{k}\right\| T U$ and $\left|A_{k} B_{k}\right|=\left|C_{k} D_{k}\right|=L_{k}=\sin k \alpha$ for every $k \in\{1,2, \ldots, 2 n-1\}$.

Denote by $d\left(A_{k} B_{k}, C_{k} D_{k}\right)=h_{k}$ the perpendicular distance between $A_{k} B_{k}$ and $C_{k} D_{k}$. Obviously, $1 \geq h_{1} \geq h_{2} \geq \cdots \geq h_{2 n-2} \geq h_{2 n-1} \geq 0$. For convenience, let $L_{0}=0$, $L_{2 n}=1 ; h_{0}=1, h_{2 n}=0$.

\section{Preliminary Lemmas}

Lemma 1. Using the notation introduced above, we have

$$
\operatorname{area}(\mathcal{C}) \leq \sin \alpha \cdot\left(1+L_{2 n-1} h_{1}+L_{2 n-2} h_{2}+\cdots+L_{2} h_{2 n-2}+L_{1} h_{2 n-1}\right) .
$$

Proof. We consider the area of the nonconvex polygon that contains $\mathcal{C}$ and has the edges parallel to $T U$ and $V W$ (see Fig. 5-this polygon is $\mathcal{C}$ plus the shaded small triangles). It follows that

$$
\begin{aligned}
\operatorname{area}(\mathcal{C}) & <\sum_{k=0}^{2 n-1} L_{k+1}\left(h_{k}-h_{k+1}\right) \\
& =\sum_{k=0}^{2 n-1}\left(L_{k+1}-L_{k}\right) h_{k}=2 \sin \frac{\alpha}{2} \cdot \sum_{k=0}^{2 n-1} h_{k} \cos \left(k+\frac{1}{2}\right) \alpha
\end{aligned}
$$




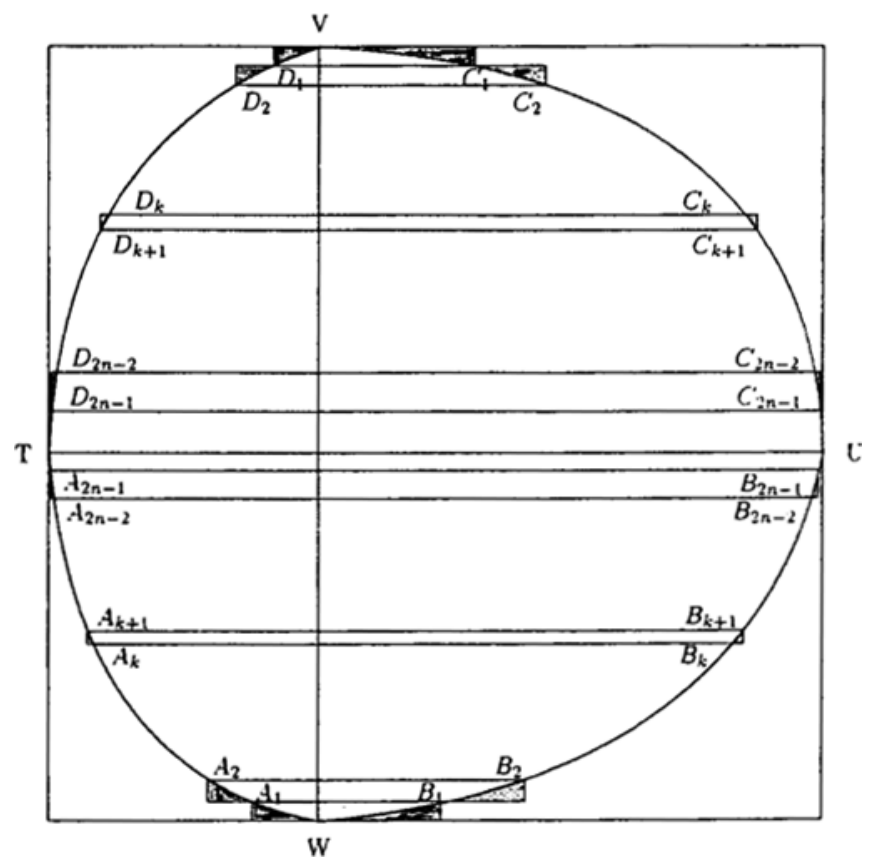

Fig. 5

$$
\begin{aligned}
& =\sin \alpha \cdot \sum_{k=0}^{2 n-1} h_{k} \cos k \alpha-2 \sin ^{2} \frac{\alpha}{2} \cdot \sum_{k=0}^{2 n-1} h_{k} \sin k \alpha \\
& <\sin \alpha \cdot \sum_{k=0}^{2 n-1} L_{2 n-k} h_{k} .
\end{aligned}
$$

Lemma 2. For every $0 \leq i \leq j \leq k \leq 2 n$ we have

$$
\left|\begin{array}{ccc}
1 & 1 & 1 \\
L_{i} & L_{j} & L_{k} \\
h_{i} & h_{j} & h_{k}
\end{array}\right| \leq 0
$$

Proof. We consider a coordinate-system with origin $O$ (the lower left corner of the circumscribed square and the positive axes as shown in Fig. 6). Triangle $A_{i} A_{j} A_{k}$ (resp. $B_{i} B_{j} B_{k}$ ) is oriented in the clockwise (resp. counterclockwise) direction, according to our system of coordinates. Therefore,

$$
\left|\begin{array}{ccc}
1 & 1 & 1 \\
x_{B_{i}} & x_{B_{j}} & x_{B_{k}} \\
y_{B_{i}} & y_{B_{i}} & y_{B_{k}}
\end{array}\right| \geq 0 \quad \text { and } \quad\left|\begin{array}{ccc}
1 & 1 & 1 \\
x_{A_{i}} & x_{A_{j}} & x_{A_{k}} \\
y_{A_{i}} & y_{A_{j}} & y_{A_{k}}
\end{array}\right| \leq 0
$$




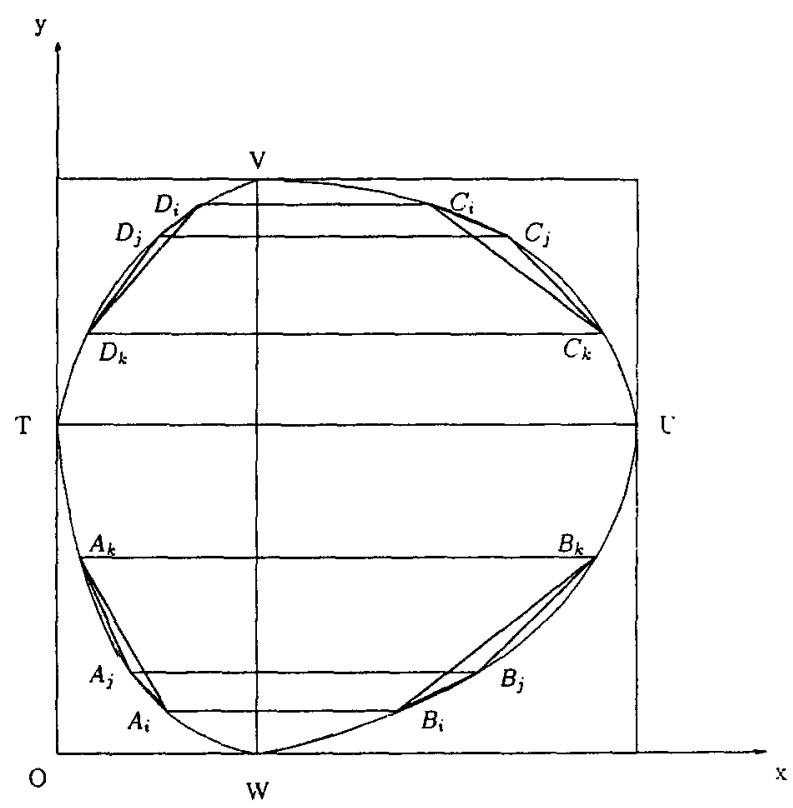

Fig. 6

However, $y_{A_{m}}=y_{B_{m}}$ for every $m \in\{i, j, k\}$, which implies that

$$
\left|\begin{array}{ccc}
1 & 1 & 1 \\
x_{B_{i}}-x_{A_{i}} & x_{B_{j}}-x_{A_{j}} & x_{B_{k}}-x_{A_{k}} \\
y_{B_{i}} & y_{B_{j}} & y_{B_{k}}
\end{array}\right| \geq 0
$$

so

$$
\left|\begin{array}{ccc}
1 & 1 & 1 \\
L_{i} & L_{j} & L_{k} \\
y_{B_{i}} & y_{B_{j}} & y_{B_{k}}
\end{array}\right| \geq 0
$$

Analogously, we obtain

$$
\left|\begin{array}{ccc}
1 & 1 & 1 \\
L_{i} & L_{j} & L_{k} \\
y_{C_{i}} & y_{C_{j}} & y_{C_{k}}
\end{array}\right| \leq 0
$$

Now subtracting inequality (3) from (4) and using $y_{C_{m}}-y_{B_{m}}=h_{m}$ for every $m \in$ $\{i, j, k\}$, we obtain the required relation.

Lemma 3. For every $0 \leq i, j \leq 2 n$ we have

$$
L_{i} h_{j}+L_{j} h_{i} \leq 1
$$

Proof. Consider the same system of coordinates as in Lemma 2 (see Fig. 7). By sym- 


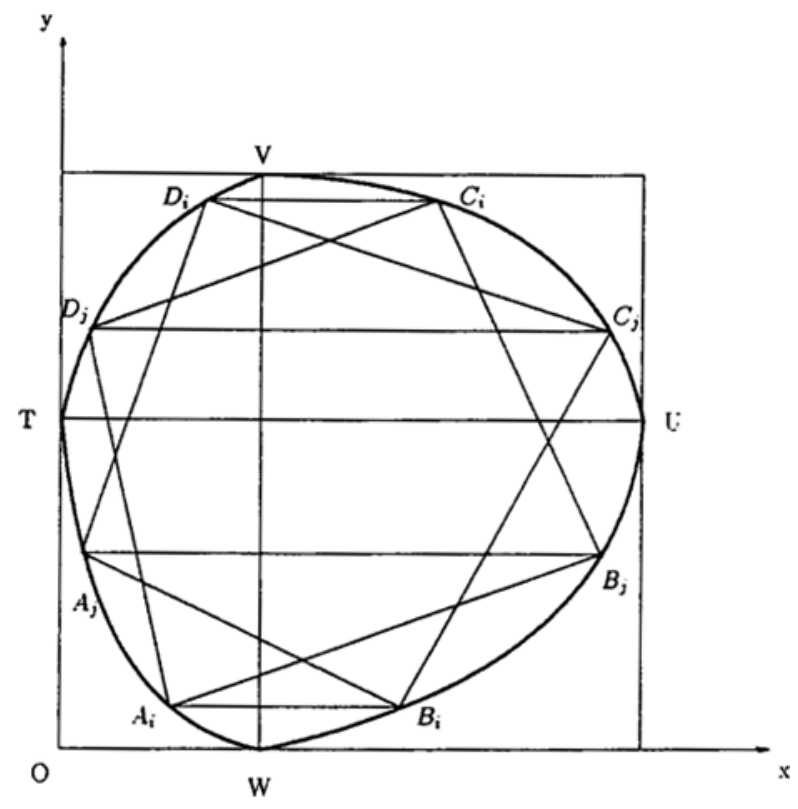

Fig. 7

metry, we can take $i \leq j$. We have that

$$
\begin{aligned}
2 \cdot \operatorname{area}\left(A_{i} B_{j} C_{i} D_{j}\right) & =\left(x_{B_{j}}-x_{D_{j}}\right)\left(y_{C_{i}}-y_{A_{i}}\right)+\left(x_{C_{i}}-x_{A_{i}}\right)\left(y_{D_{j}}-y_{B_{j}}\right) \\
& =\left(x_{B_{j}}-x_{D_{j}}\right) h_{i}+\left(x_{C_{i}}-x_{A_{i}}\right) h_{j} .
\end{aligned}
$$

Analogously, we obtain

$$
2 \operatorname{area}\left(A_{j} B_{i} C_{j} D_{i}\right)=\left(x_{C_{j}}-x_{A_{j}}\right) h_{i}+\left(x_{B_{i}}-x_{D_{i}}\right) h_{j} .
$$

The last two relations imply that

$$
\operatorname{area}\left(A_{i} B_{j} C_{i} D_{j}\right)+\operatorname{area}\left(A_{j} B_{i} C_{j} D_{i}\right)=L_{i} h_{j}+L_{j} h_{i} .
$$

Since every quadrilateral inscribed in $\mathcal{C}$ has area at most $\frac{1}{2}$ (as we have seen in Section 2), the required relation follows.

Return to Figure 5. We are interested in $p$-hexagons belonging to direction $T U$. There are two types of such $p$-hexagons:

Type 1: $T A_{i} B_{i} U C_{i} D_{i}, i \in\{0,1, \ldots, 2 n-1\}$,

$$
\operatorname{area}\left(T A_{i} B_{i} U C_{i} D_{i}\right)=\frac{1}{2} h_{i}\left(1+L_{i}\right) .
$$

Type 2: $A_{i} W B_{i} C_{i} V D_{i}, i \in\{1,2, \ldots, 2 n\}$,

$$
\operatorname{area}\left(A_{i} W B_{i} C_{i} V D_{i}\right)=\frac{1}{2}\left(1+h_{i}\right) L_{i} .
$$


Denote

$$
\begin{aligned}
& h_{p}\left(1+L_{p}\right)=\max _{0 \leq i \leq 2 n-1} h_{i}\left(1+L_{i}\right), \\
& \left(h_{q}+1\right) L_{q}=\max _{1 \leq i \leq 2 n}\left(1+h_{i}\right) L_{i},
\end{aligned}
$$

and

$$
2 S=\max \left\{h_{p}\left(1+L_{p}\right),\left(1+h_{q}\right) L_{q}\right\}
$$

Hence, if $H$ denotes the $p$-hexagon of maximum area belonging to direction $T U$, we obtain $\operatorname{area}(H) \geq S$. We need another lemma.

Lemma 4. For every $k \in\{1,2, \ldots, 2 n-1\}$,

$$
h_{k} \leq \frac{2 S\left(1+2 L_{p}-L_{k}\right)}{\left(1+L_{p}\right)^{2}}+O\left(\frac{1}{n}\right)
$$

and

$$
h_{k} \leq \frac{2 S\left(2 L_{q}-L_{k}\right)}{L_{q}^{2}}-1+O\left(\frac{1}{n}\right)
$$

Proof. First suppose $k \leq p$. Using Lemma 2 for the triplet $(k, p-1, p)$, it follows that

$$
h_{k}\left(L_{p}-L_{p-1}\right)+h_{p}\left(L_{p-1}-L_{k}\right) \leq h_{p-1}\left(L_{p}-L_{k}\right) .
$$

However, (6) implies that $h_{p-1} \leq h_{p}\left(1+L_{p}\right) /\left(1+L_{p-1}\right)$.

After some straightforward calculations we obtain

$$
h_{k} \leq \frac{h_{p}\left(1+L_{p}+L_{p-1}-L_{k}\right)}{1+L_{p-1}} \leq \frac{2 S\left(1+L_{p}+L_{p-1}-L_{k}\right)}{\left(1+L_{p-1}\right)\left(1+L_{p}\right)} .
$$

We can easily check that

$$
h_{k} \leq \frac{2 S\left(1+2 L_{p}-L_{k}\right)}{\left(1+L_{p}\right)^{2}}+O\left(\frac{1}{n}\right)
$$

Analogously, if we use Lemma 2 for the triplet $(p, p+1, k)$, where $k \geq p+1$, and $h_{p+1}\left(1+L_{p+1}\right) \leq h_{p}\left(1+L_{p}\right)$, the same type of calculations gives

$$
h_{k} \leq \frac{2 S\left(1+L_{p}+L_{p+1}-L_{k}\right)}{\left(1+L_{p+1}\right)\left(1+L_{p}\right)}=\frac{2 S\left(1+2 L_{p}-L_{k}\right)}{\left(1+L_{p}\right)^{2}}+O\left(\frac{1}{n}\right) .
$$

To prove (10), it would be sufficient to repeat the above procedure for the triplets $(k, q, q+1)$ if $k \leq q$, and $(q, q+1, k)$ if $k \geq q+1$. 


\section{Proof of the Theorem}

Our goal is to estimate area $(\mathcal{C})$ in terms of $L_{p}, L_{q}$, and $S$. Consider $x_{0}=0.2594296 \ldots$, the real root of the equation $\cos x-x=\sqrt{2} / 2$, and let $t=\left\lfloor x_{0} / \alpha\right\rfloor=\left\lfloor 4 n x_{0} / \pi\right\rfloor$. Note that $t \leq n$. We use the following notation:

$$
\begin{aligned}
& T_{1}=1+\sum_{k=1}^{t}\left(L_{2 n-k} h_{k}+L_{k} h_{2 n-k}\right), \\
& T_{2}=\sum_{k=t+1}^{n} L_{2 n-k} h_{k}, \\
& T_{3}=\sum_{k=n+1}^{2 n-t-1} L_{2 n-k} h_{k} .
\end{aligned}
$$

Obviously,

$$
1+\sum_{k=1}^{2 n-1} L_{2 n-k} h_{k}=T_{1}+T_{2}+T_{3} .
$$

Inequality (1) and these last relations imply that

$$
\operatorname{area}(\mathcal{C}) \leq \sin \alpha \cdot\left(T_{1}+T_{2}+T_{3}\right) .
$$

Lemma 3 implies $L_{2 n-k} h_{k}+L_{k} h_{2 n-k} \leq 1$ for every $k \in\{1,2, \ldots, 2 n-1\}$. Therefore,

$$
\begin{aligned}
\sin \alpha \cdot T_{1} & \leq \sin \alpha \cdot\left(1+\sum_{k=1}^{t} 1\right)=\sin \alpha \cdot(t+1) \\
& =\left[\alpha+O\left(\frac{1}{n^{2}}\right)\right] \cdot\left[\frac{x_{0}}{\alpha}+o\left(\frac{1}{n}\right)\right] \\
& =\left[\frac{\pi}{4 n}+O\left(\frac{1}{n^{2}}\right)\right] \cdot\left[\frac{4 n}{\pi} x_{0}+O\left(\frac{1}{n}\right)\right] \\
& =x_{0}+O\left(\frac{1}{n}\right) .
\end{aligned}
$$

Thus,

$$
\sin \alpha \cdot T_{1} \leq x_{0}+O\left(\frac{1}{n}\right) .
$$

In order to estimate the other two quantities from (11), we need the following.

\section{Lemma 5.}

$$
\begin{aligned}
& \sin \alpha \cdot \sum_{k=t+1}^{n} L_{2 n-k}=\frac{\sqrt{2}}{2}-\sin x_{0}+O\left(\frac{1}{n}\right), \\
& \sin \alpha \cdot \sum_{k=t+1}^{n} L_{2 n-k} L_{k}=\cos \frac{2 x_{0}}{4}+O\left(\frac{1}{n}\right),
\end{aligned}
$$




$$
\begin{aligned}
& \sin \alpha \cdot \sum_{k=n+1}^{2 n-t-1} L_{2 n-k} L_{k}=\frac{\cos 2 x_{0}}{4}+O\left(\frac{1}{n}\right), \\
& \sin \alpha \cdot \sum_{k=n+1}^{2 n-t-1} L_{2 n-k}=\cos x_{0}-\frac{\sqrt{2}}{2}+O\left(\frac{1}{n}\right) .
\end{aligned}
$$

Proof. Some simple calculations give that

$$
\begin{aligned}
\sin \alpha \cdot \sum_{k=t+1}^{n} L_{2 n-k} & =\frac{\cos (\pi / 4)+\cos (\pi / 4-\alpha)}{2}-\frac{\sin (t+1) \alpha+\sin t \alpha}{2} \\
& =\frac{\sqrt{2}}{2}-\sin x_{0}+O\left(\frac{1}{n}\right) \\
\sin \alpha \cdot \sum_{k=t+1}^{n} L_{2 n-k} L_{k} & =\frac{\cos (2 t+1) \alpha+\sin \alpha}{4}=\frac{\cos 2 x_{0}}{4}+O\left(\frac{1}{n}\right), \\
\sin \alpha \cdot \sum_{k=n+1}^{2 n-t-1} L_{2 n-k} L_{k} & =\frac{\cos (2 t+1) \alpha-\sin \alpha}{4}=\frac{\cos 2 x_{0}}{4}+O\left(\frac{1}{n}\right) .
\end{aligned}
$$

Finally,

$$
\begin{aligned}
\sin \alpha \cdot \sum_{k=n+1}^{2 n-t-1} L_{2 n-k} & =\frac{\cos (t+1) \alpha+\cos t \alpha}{2}-\frac{\sin (\pi / 4)+\sin (\pi / 4-\alpha)}{2} \\
& =\cos x_{0}-\frac{\sqrt{2}}{2}+O\left(\frac{1}{n}\right)
\end{aligned}
$$

Now,

$$
\sin \alpha \cdot T_{2}=\sin \alpha \cdot \sum_{k=i+i}^{n} L_{2 n-k} h_{k} .
$$

Using relation (9), it follows that

$$
\begin{aligned}
\sin \alpha \cdot T_{2} \leq & \sin \alpha \cdot\left[\sum_{k=t+1}^{n} L_{2 n-k}\left(\frac{2 S\left(1+2 L_{p}-L_{k}\right)}{\left(1+L_{p}\right)^{2}}+O\left(\frac{1}{n}\right)\right)\right] \\
= & \sin \alpha \cdot\left[\frac{2 S}{\left(1+L_{p}\right)^{2}} \cdot \sum_{k=t+1}^{n} L_{2 n-k}\left(1+2 L_{p}-L_{k}\right)+O(1)\right] \\
= & \frac{2 S\left(1+2 L_{p}\right)}{\left(1+L_{p}\right)^{2}} \cdot \sin \alpha \cdot \sum_{k=t+1}^{n} L_{2 n-k}-\frac{2 S}{\left(1+L_{p}\right)^{2}} \cdot \sin \alpha \\
& \sum_{k=t+1}^{n} L_{2 n-k} L_{k}+O\left(\frac{1}{n}\right) .
\end{aligned}
$$


Now, using equalities (13), (14), and (15) we obtain

$$
\begin{aligned}
\sin \alpha \cdot T_{2} \leq & \frac{2 S\left(1+2 L_{p}\right)}{\left(1+L_{p}\right)^{2}} \cdot\left(\frac{\sqrt{2}}{2}-\sin x_{0}+O\left(\frac{1}{n}\right)\right) \\
& -\frac{2 S}{\left(1+L_{p}\right)^{2}} \cdot\left(\frac{\cos 2 x_{0}}{4}+O\left(\frac{1}{n}\right)\right)+O\left(\frac{1}{n}\right) .
\end{aligned}
$$

Hence,

$$
\sin \alpha \cdot T_{2} \leq \frac{2 S\left(1+2 L_{p}\right)}{\left(1+L_{p}\right)^{2}} \cdot\left(\frac{\sqrt{2}}{2}-\sin x_{0}\right)-\frac{S \cos 2 x_{0}}{2\left(1+L_{p}\right)^{2}}+O\left(\frac{1}{n}\right) .
$$

On the other hand, using (10) we obtain

$$
\begin{aligned}
& \sin \alpha \cdot T_{3}=\sin \alpha \cdot \sum_{k=n+1}^{2 n-t-1} L_{2 n-k} h_{k} \\
& \leq \sin \alpha \cdot\left[\sum_{k=n+1}^{2 n-t-1} L_{2 n-k} \cdot\left(\frac{2 S\left(2 L_{q}-L_{k}\right)}{L_{q}^{2}}-1+O\left(\frac{1}{n}\right)\right)\right] \\
&=\sin \alpha \cdot\left[\frac{4 S}{L_{q}} \cdot \sum_{k=n+1}^{2 n-t-1} L_{2 n-k}-\frac{2 S}{L_{q}^{2}} \cdot \sum_{k=n+1}^{2 n-t-1} L_{2 n-k} L_{k}\right. \\
&\left.-\sum_{k=n+1}^{2 n-t-1} L_{2 n-k}+O(1)\right]
\end{aligned}
$$

Now (14), (15), and (16) imply that

$$
\begin{aligned}
\sin \alpha \cdot T_{3} \leq & \frac{4 S}{L_{q}} \cdot\left(\cos x_{0}-\frac{\sqrt{2}}{2}+O\left(\frac{1}{n}\right)\right)-\frac{2 S}{L_{q}^{2}} \cdot\left(\frac{\cos 2 x_{0}}{4}+O\left(\frac{1}{n}\right)\right) \\
& -\left(\cos x_{0}-\frac{\sqrt{2}}{2}\right)+O\left(\frac{1}{n}\right) .
\end{aligned}
$$

Therefore,

$$
\sin \alpha \cdot T_{3} \leq \frac{4 S}{L_{q}} \cdot\left(\cos x_{0}-\frac{\sqrt{2}}{2}\right)-\frac{S \cos 2 x_{0}}{2 L_{q}^{2}}-\left(\cos x_{0}-\frac{\sqrt{2}}{2}\right)+O\left(\frac{1}{n}\right) .
$$

From (11), (12), (17), and (18) we obtain that

$$
\begin{aligned}
\operatorname{area}(\mathcal{C}) \leq & \left(x_{0}+\frac{\sqrt{2}}{2}-\cos x_{0}\right)+\frac{2 S\left(1+2 L_{p}\right)}{\left(1+L_{p}\right)^{2}} \cdot\left(\frac{\sqrt{2}}{2}-\sin x_{0}\right)-\frac{S \cos 2 x_{0}}{2\left(1+L_{p}\right)^{2}} \\
& +\frac{4 S}{L_{q}} \cdot\left(\cos x_{0}-\frac{\sqrt{2}}{2}\right)-\frac{S \cos 2 x_{0}}{2 L_{q}^{2}}+O\left(\frac{1}{n}\right) .
\end{aligned}
$$


Finally,

$$
\begin{aligned}
\frac{\operatorname{area}(\mathcal{C})}{S} \leq & \frac{\left(1+2 L_{p}\right)}{\left(1+L_{p}\right)^{2}} \cdot\left(\sqrt{2}-2 \sin x_{0}\right)-\frac{\cos 2 x_{0}}{2\left(1+L_{p}\right)^{2}} \\
& +\frac{2}{L_{q}} \cdot\left(2 \cos x_{0}-\sqrt{2}\right)-\frac{\cos 2 x_{0}}{2 L_{q}^{2}}+O\left(\frac{1}{n}\right)
\end{aligned}
$$

It is now easy to check that

$$
\frac{\left(1+2 L_{p}\right)}{\left(1+L_{p}\right)^{2}} \cdot\left(\sqrt{2}-2 \sin x_{0}\right)-\frac{\cos 2 x_{0}}{2\left(1+L_{p}\right)^{2}} \leq \frac{4\left(\sqrt{2}-2 \sin x_{0}\right)}{4+\sqrt{2}+2 \sin x_{0}}
$$

and equality occurs iff $L_{p}=\left(\sin x_{0}\right) / 2+\sqrt{2} / 4=0.481818 \ldots$ Also,

$$
\frac{2}{L_{q}} \cdot\left(2 \cos x_{0}-\sqrt{2}\right)-\frac{\cos 2 x_{0}}{2 L_{q}^{2}} \leq \frac{4 x_{0}}{x_{0}+\sqrt{2}}
$$

and equality occurs iff $L_{q}=\left(\cos x_{0}\right) / 2+\sqrt{2} / 4=0.8368216 \ldots$ Hence,

$$
\frac{\operatorname{area}(\mathcal{C})}{S} \leq \frac{4\left(\sqrt{2}-2 \sin x_{0}\right)}{4+\sqrt{2}+2 \sin x_{0}}+\frac{4 x_{0}}{x_{0}+\sqrt{2}}+O\left(\frac{1}{n}\right)=1.2281771 \ldots
$$

This final relation proves the theorem.

This result is nearly the best that can be obtained using only the ideas presented above. Consider $\mathcal{C}$ as shown in Fig. 8. $T U$ and $V W$ are two perpendicular diameters of the circle

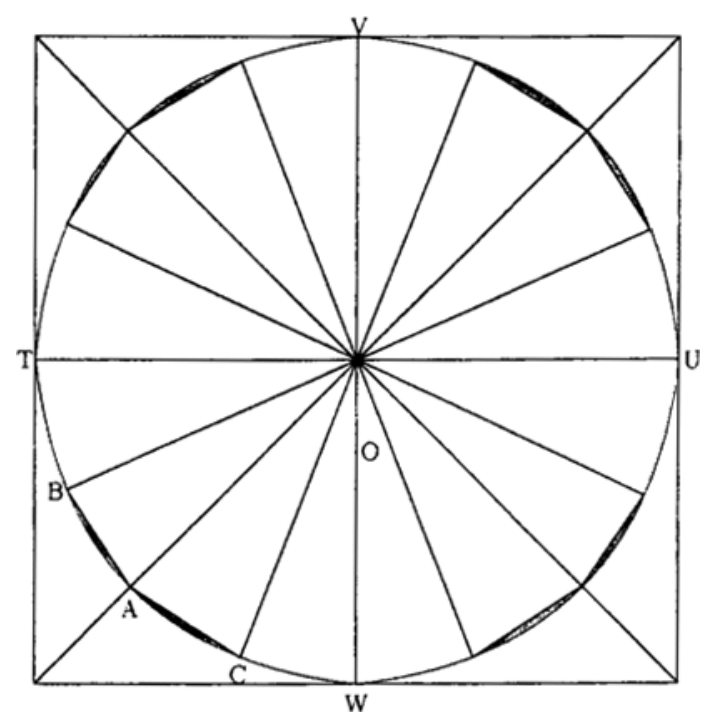

Fig. 8 
of radius $\frac{1}{2}$. Point $A$ is the middle of the arc $T W$ and the points $B$ and $C$ are chosen so that the measure of the small angles $\widehat{T O B}$ and $\widehat{C O W}$ are equal to $x_{0}=0.2594296 \ldots$ Repeat this construction in the other three quadrants and then take $\mathcal{C}$ to be the circular disk from which we take off those eight shaded segments of the circle. Obviously, $T V U W$ is the quadrilateral of maximum area from all quadrilaterals inscribed in $\mathcal{C}$.

Some straightforward calculations show that

$$
\operatorname{area}(\mathcal{C})=\left(\frac{\sqrt{2}}{2}+1\right) \cos x_{0}-\frac{\sqrt{2}}{2}\left(1+\sin x_{0}\right)=0.7614804 \ldots
$$

On the other hand, it is not difficult to check that all $p$-hexagons belonging to direction $T U$ have area at most

$$
\operatorname{area}(H) \leq \frac{\left[(\sqrt{2}+1) \cos x_{0}-1-\sin x_{0}\right]^{2}}{4\left(2 \cos x_{0}-\sqrt{2}\right)\left(\sqrt{2}-2 \sin x_{0}\right)}=0.6200668 \ldots
$$

Consequently, it follows that

$$
\frac{\operatorname{area}(\mathcal{C})}{\operatorname{area}(H)} \geq \frac{0.7614804}{0.6200668}=1.2280619 \ldots
$$

This bound is not far from the one obtained in the theorem.

\section{References}

1. C. H. Dowker, On minimum circumscribed polygons, Bull. Amer. Math. Soc., 50 (1944), 120-122.

2. R. Kershner, The number of circles covering a set, Amer. J. Math., 61 (1939), 665-671.

3. W. Kuperberg, Covering the plane with copies of a convex body, Bult. London Math. Soc., 21 (1989), 82-86.

4. J. Pach and P. K. Agarwal, Combinatorial Geometry, Wiley-Interscience, New York, 1995.

5. E. Sas, Über eine Extremumeigenschaft der Ellipsen, Compositio Math., 6 (1939), 468-470.

Received June 1, 1996, and in revised form January 24, 1997. 